\title{
Produtos dos cuidados de enfermagem
}

\author{
Products of nursing care \\ Productos de los cuidados de enfermería
}

Maria José Coelho'
'Universidade Federal do Rio de Janeiro. Escola de Enfermagem Anna Nery. Rio de Janeiro, RJ

Submissão: 19/08/2008

Aprovação: 29/10/2009

\section{RESUMO}

A ideia é pensar nos conhecimentos e saberes do ato de cuidar em enfermagem nas tecnologias, no processo saúde-doença e seus determinantes e o cuidado enquanto produto. De 1980 a 1992, houve 544.357 leitos ocupados e 19.6 milhões de internações; logo, 544.357 ou 19.6 milhões de cuidados prestados. A reflexão emergiu no Grupo de Pesquisa Cuidar Cuidados de Enfermagem / Escola de Enfermagem Anna Nery subsidiada por conceitos de cotidiano de Certeau, de cuidar, cuidado, cliente, na análise dos elementos constitutivos dos Cuidados de Enfermagem. A construção de conhecimentos e saberes apontam a criação de produtos como livros/ capítulos, protocolos, jornal, agenda, DVDs, CDs, teses, dissertações etc. As maneiras de cuidar proporcionam a criação de produtos, numa construção tecida diariamente.

Descritores: Enfermagem; Cuidados de enfermagem; Conhecimento.

\section{ABSTRACT}

The idea is to think about the knowledge and the learning involved in nursing care, its technologies, the health-disease process and its determinants, as well as the care as a product. From 1980 to 1992, 544.357 hospital beds were occupied and 19,6 million hospitalizations; therefore, 544,357 or 19.6 million of delivered care. This consideration emerged from the Research Group Care/Nursing Care from the Anna Nery Nursing School subsidized by Certeau's concepts of daily care and concepts of care practices, care, and client, for the analysis of the constitutive elements in nursing care. The knowledge and learning construction points to the creation of products such as books/ chapters, protocols, new papers, agendas, DVDs, CDs, thesis, and dissertations. The way of caring contribute to the development of a series of products, built on a daily basis.

Descriptors: Nursing; Nursing care; Knowledge.

\section{RESUMEN}

La idea es pensar en los conocimientos y en las sabidurías del acto de cuidar en enfermería, en las tecnologías, en el proceso saludenfermedad y sus determinantes y en el cuidado en cuanto producto. De 1980 a 1992, había 544.357 camas ocupadas y 19,6 milliones de internaciones; esto significa 544.357 o 19,6 milliones de cuidados prestados. La reflexión surgió en el grupo de pesQuisa Cuidar/ cuidados de Enfermería de la Escuela de Enfermería Anna Nery, subsidiado por los conceptos del cotidiano de Certeau y de cuidar, cuidado y cliente en la análisis de los elementos constitutivos de los cuidados de Enfermería. La construcción de los conocimientos apuntan la creación de productos, como libros/capítulos, protocolos, periódicos, agenda, DVDs, CDs, tesis, disertaciones, y las maneras de cuidar proporcionan la creacón de productos, en una construcción tejida diariamente.

Descriptores: Enfermería; Atención de enfermería; Conocimiento. 


\section{CONSIDERAÇÕES INICIAIS}

A idea desse artigo de reflexão é pensar na produção de conhecimentos e saberes sobre o ato de cuidadar em enfermagem, levando em consideração a relação com o cuidar e os cuidados de enfermagem, as novas tecnologias, o processo saúde-doença e seus determinantes para o cliente enfermo hospitalizado e re-internado com doenças crônicas ou agudas várias vezes, no sentido de criar produtos a partir dos cuidados de enfermagem prestados.

Há muitos anos, no inicio da década de 1980, Quando comecei, ainda recém-formada, a cuidar no Hospital Universitário Clementino Fraga Filho da UFRJ, observei, no cotidiano de cuidados, Que a clientela era receptora direta dos produtos, sejam eles criados e/ ou renovados, construídos ou improvisados pela indústria e dados como ferramenta para a elaboração dos cuidados, ou mesmo aqueles Que emergem do cotidiano nos Quais denominamos "improvisação"; de outro lado, os profissionais criadores ou usuários dos produtos.

Poucas vezes, ouvi a correlação dessa criação feita no silêncio do cotidiano como algo Que pudesse ser considerado criação própria e legítima de enfermagem, dos enfermeiros ou de um dos seus integrantes.

As improvisações - recriações - podiam e podem ser encontradas nas Instituições de Saúde, mas o autor sempre é desconhecido. Um evento criado hoje se propaga por todas as instituições numa testagem contínua via divulgação boca-a-boca, de forma oralista.

Lembro de muitas enfermeiras costurando em suas casas um saco de tecido de algodão preto para proteger as soluções sensíveis à luz na administração de drogas. Hoje, mais de 30 anos após, há equipo para soluções hipersensíveis à luminosidade.

Congresso vai congresso vêm, encontros vão encontros vêm, e lá está uma ou outra enfermeira, diga-se de passagem, a pioneira, a inovadora, a apresentar as suas criações, os seus inventos. Todos nós a admiramos e até pensamos em adotá-los no dia-a-dia do cuidado prestado aos clientes.

Mas, Quando voltamos para a prática, continuamos a usar o Que nos é oferecido, geralmente por uma grande empresa via vários setores da gestão de saúde Institucional e, muitas vezes, sem aderência direta aos cuidados prestados ao cliente. Quando olhamos uma criação em enfermagem, seja ela Qual for, não podemos deixar de correlacioná-la aos clientes. No meu caso, penso logo naqueles clientes hospitalizados, no trajeto dos cuidados Que entra com ele na admissão e vai até o momento da alta, como é mostrado a seguir, e no Que foi criado, testado, aprovado ou reprovado durante esse caminho Que ficou encoberto pelo silêncio do Cotidiano.

O caminho de (re)criação desses produtos começa Quando o cliente se registra e é admitido no Serviço de Admissão, Setor ou Enfermaria seguindo um caminho ${ }^{(1)}$ no Qual emerge vários cuidados.

- Cliente/Paciente

- Recebido no local de Internação

- Coleta de dados para o histórico de Enfermagem

- Implementação dos primeiros cuidados hospitalares

- Atendimento médico, vigilância nos cuidados de Enfermagem de compartilhamento com outros profissionais

- Implementações de Cuidados de Enfermagem Complexos e de altíssima complexidade no Cuidar individual ou coletivo

- Requisição de exames/ resultados/ encaminhamentos/ instituição de tratamento e conseQüentes cuidados referentes a estes/ Implementação/ criação/ improvisação de cuidados

- A luta no limiar entre a vida e a morte - Cuidados na fronteira do limite

- Em vigia e vigilância intensivas e seqüenciais dos cuidados de Enfermagem

- Cuidar no Encaminhamento para o CTI / Centro Cirúrgico ou Setor de Internação

- Cuidar no momento da morte/óbito

- Cuidar do corpo morto

- Encaminhar o corpo morto à patologia/ cuidar no tocante às providências legais Quanto ao corpo encaminhado ao Instituto médico legal, se for o caso.

- Cuidar da Família/amigos/companheiros/conhecidos, entre outros... (1)

(Re)Criação diária e intensa da cliente/paciente Que entra e sai do processo saúde/doença/cuidado e Que perpassa todo o fluxograma.

\section{REFLEXÃO}

Refletindo um pouco mais Quanto aos produtos oriundos dos cuidados, percebemos Que, de 1980 a 1992, no Brasil, houve 544.357 leitos hospitalares ocupados e 19,6 milhões de internação ${ }^{(2)}$, e numa projeção podemos afirmar Que ocorreram 544.357 cuidados ou 19,6 milhões de cuidados de Enfermagem. Poderíamos pensar, também, na ocorrência de 544.357 produtos desses cuidados.

Observando com mais profundidade, percebemos que se passou uma década; logo, essa projeção já se modificou em números e circunstâncias, mas e os produtos oriundos e emergentes dos cuidados de enfermagem? O Que aconteceu durante essa década? Qual o seu perfil? Onde andam? Quem os consome?

Podemos avançar mais o ponto de reflexão ao analisar os elementos constitutivos do Cuidar e dos Cuidados de Enfermagem, como produtos a serem utilizados pela população da América Latina projetada para 2025, de 436,21 2 milhões de pessoas, e pelo Brasil de hoje, de Quase 200 milhões de pessoas ${ }^{(3)}$.

Construir produtos oriundos dos cuidados em enfermagem é necessário, assim como construir redes de conhecimento e conhecimentos em rede, seja Qual for a sua natureza.

Essa reflexão dos produtos de Enfermagem novos ou renovados versus população cuidada pela Enfermagem através dos cuidados emerge semanalmente no Grupo de Pesquisa Cuidar / Cuidados de Enfermagem / Núcleo de Pesquisa Enfermagem Hospitalar / Departamento de Enfermagem Médico-Cirúrgica Que possui reuniões periódicas, com um banco de dados de textos relacionados ao Cuidar e aos Cuidados de Enfermagem, e aulas para mestres, doutores e alunos especiais da disciplina Seminário Assistencial com ênfase no cuidar e nos cuidados de Enfermagem EEAN/UFRJ, desde 1999. Mas Quando emerge, há um discurso recorrente de (in)visibilidade desses cuidados prestados assim como de sua criação.

Outro ponto freQüentemente recorrente é no tocante à orientação. Essa expressão vem acompanhada Quase sempre de Que o cliente/ paciente não conhece tais sinais ou sintomas ou patologia a ele atribuída. Na contra-argumentação, destacamos, mas ele não deve conhecer e sim ser informado, Quem deve conhecer é o profissional 
de enfermagem, elaborador e construtor desse cuidado via ciência. Quando usamos apenas a expressão oral para transmitir alguma coisa, geralmente não obtemos êxito ou esse êxito é parcial. Mas por Que não lhe damos um produto oriundo desse cuidado oral para Que o mesmo possa usar? Um produto Que tenha embutido em seu interior a orientação, um produto oriundo desse momento de encontro oralista entre enfermagem/cliente ou cliente-enfermagem.

Para subsidiar essa reflexão cotidiana cuidados como produtos ou produtos como cuidados dentro e fora do Grupo de pesquisa ou nas disciplinas, ou nas conversas de corredores dos Hospitais em especial, recorremos sempre aos conceitos de cotidiano de Certeau ${ }^{(4)}$, Que diz "extrair do seu ruído as maneiras de fazer"; os de Coelho et al ${ }^{(1)}$ de cuidar como processo de expressão, de reflexão, de elaboração do pensamento, de imaginação, de meditação e de aplicação intelectual [...]"; cuidados "ação imediata prestada [...], em curto espaço de tempo, desenvolvido em vários momentos, envolvendo segurança e competência, aliadas à tecnologia especifica Que a situação exige" e cliente "aquele que sofreu uma violência ou acidente físico e/ou orgânico, e que traz consigo as suas características pessoais, familiares e sociais, necessitando urgentemente, do cuidar/cuidados de enfermagem [...]"; dos princípios de conservação de Levine ${ }^{(1)}$ Que considera como tema central a ação de enfermagem baseada no cuidado total da pessoa e em princípios, significando: a) manter o equilibrio, com enfouue nos aspectos da energia: repouso, nutrição e exercício adequados, integridade; b) estrutural: o corpo e o seu funcionamento, manutenção e recuperação da estrutura do corpo, prevenção do colapso físico e promoção da cura; c) pessoal: manutenção e recuperação da identidade e auto-estima do cliente; d) social: reconhecê-lo como um ser social e do processo de enfermagem de Horta ${ }^{(1)}$, onde a criação dos produtos perpassa todas as fases desse processo na busca do cuidar e dos cuidados prestados aos clientes enfermos hospitalizados e re-hospitalizados com doenças crônicas e agudas, numa perspectiva de criar um produto.

A discussão dos produtos de enfermagem tem a sua construção nos conhecimentos e saberes Que apontam para a criação( ${ }^{(1,5-8)}$ de livros, capítulos, protocolos de cuidados, jornal do cuidado, agenda do cuidado, DVDS, CDs, fitas de vídeos, teses, dissertações, monografias, cartazes, curativos, esponjas, garotes entre tantos produtos Que os cuidados proporcionam criar e são tão pouco conhecidos pela Enfermagem Que, no Brasil, fica em torno de 1.026 .000 (hum milhão e 26 mil) profissionais ${ }^{(9)}$.

Diante dessas observações e reflexões, em Que os produtos criados podem ser consumidos pela população de profissional e sua clientela de forma rápida e eficaz, podemos acrescentar Que para isso é necessário alguns pontos para uma agenda de discussão:

- Ousadia

- PesQuisas convencionais e não convencionais

- Gerar idéias Que se transformam em novas idéias

- Considerar que os erros geram aprendizagem

- Inocular ambiente de entusiasmo

- Debater novas idéias - brainstorms

- Implementar uma Educação empreendedora em enfermagem

- Criar Rede de Cuidados de Enfermagem Nacional e Internacional

- Aprender a conviver com as novidades

- Criar espaços nos Eventos nos Eventos Cientificos

- Fortalecer a Rede de Idéias cuidados enquanto produtos

- Fortalecer a Interatividade
- Bate-papo de cuidados

- Inovações do Cuidar

- Procedimento

- Pensar em Tecnologias

- Pensar em Transferência de Tecnologia e Comercialização

- Pensar em Propriedade Industrial

- Se aproximar da Academia Mundial de Propriedade Intelectual da Organização mundial de Propriedade Intelectual (OMPI) e

- Do Escritório de Assistência Técnica e Capacitação para América Latina e Caribe da Organização Mundial da Propriedade Intelectual (OMPI)

- Pensar em Patente (um contrato que concede o seu detentor o direito de ser dono com exclusividade durante um período de tempo)

\section{- Escritório de Direitos Autorais}

O conhecimento assim produzido e transformado em produtos dos cuidados representa um patrimônio para a Enfermagem Brasileira como profissão, mas também para a humanidade. Os países sul-americanos como Argentina, Bolívia, Brasil, Chile, Colômbia, Equador, Paraguai, Peru, Uruguai, Venezuela tinham em 2005, 367,6 milhões de habitantes (2005) e 436,2 I 2 população projetada para $2025^{(10)}$ e enfrenta serias situações de saúde/doença de cunho multifatorial.

As maneiras de cuidar do cliente proporcionam a criação de produtos e uma Mestiçagem de saberes, pois, ao criar, há uma construção de saberes tecidos a partir de contribuições universais. Esses conhecimentos partem da mestiçagem de contribuições da física, Química, sociologia, antropologia, entre outras e de contradições da vida cotidiana.

Poucos enfermeiros migram da atividade científica para as de inovação e desenvolvimento tecnológico, gerando frágil desempenho na geração de produtos de enfermagem.

Observamos em alguns projetos políticos pedagógicos dos 630 cursos de graduação em Enfermagem na modalidade de bacharelado $^{(\text {III) }}$, a introdução de disciplinas ligadas ao empreendedorismo assim como cursos de enfermagem dentro de IES com perfil tecnológico.

\section{CONSIDERAÇÕES FINAIS}

Hoje, nós temos no Brasil, segundo o Conselho Federal de Enfermagem $^{(9)}$, 137.896 enfermeiros; 309.111 técnicos de enfermagem; 553.354 auxiliares de enfermagem e 23.623 atendentes.

No Rio de Janeiro, 19.275 enfermeiros, dos Quais 11.845 estão na cidade do Rio de Janeiro (COREn/RJ) e em torno de 150 doutores, é pouco, mas já podemos começar a criar produtos para a população carioca Que hoje está em torno de 12 milhões de pessoas e graves problemas de saúde e sociais, assim como pensar no Brasil com seus Quase 200 milhões de habitantes e um parque de idéias de Enfermagem em torno de Quase hum milhão de trabalhadores de enfermagem Que diariamente criam algo.

Vamos criar produtos dos cuidados, para Que o cliente possa usar Quando estiver em seu momento de individualidade, fazendo com Que a Enfermagem possa estar visível mesmo Quando ela acredita esta invisível. Ainda existe um caminho longo a ser percorrido, mas os resultados mostram que estamos evoluindo e que 
o cenário é muito promissor.

É preciso estimular a consciência crítica-criativa e de solução para os problemas em relação à Ciência e Tecnologia desde o curso de auxiliar de enfermagem até o curso Pós-Doutorado, pois a Criatividade é imprescindível para fazer pesquisas e interagir com a indústria e empresas. Com base nessa premissa, podemos contribuir para Que o Brasil possa subir no ranking mundial na área de pesQuisa. A posição atual é o $17^{\circ}$ lugar.

Quando li o artigo de atualização da REBEn "Incubadora de aprendizagem $[\ldots]^{\prime(12)}$, pensei logo: se há incubadora de aprendizagem haverá em breve incubadora de produtos que atenderam as duas principais clientelas: clientes de enfermagem e profissional de enfermagem.

O Brasil dispõe atualmente de 42 cursos de pós-graduação em enfermagem, sendo 26 de mestrado, 13 de doutorado e três de mestrado profissional. Os hospitais públicos e privados credenciados no Brasil são cerca de 5.000 , totalizando cerca de 400 mil leitos e 12 milhões de internação/ano ${ }^{(13)}$. Diante desse universo, temos condições e ambiente de criatividade para oferecer um produto para a clientela de Enfermagem a ser usado Quando as suas necessidades humanas básicas estiverem afetadas, deixando para as próximas décadas outros desafios emergentes das criações atuais.

A Enfermagem do Século XXI é de divulgação cientifica, tecnológica e de inovação, é o futuro que tanto esperamos hoje.

\section{REFERÊNCIAS}

I. Coelho MJ, Figueiredo NMA, Carvalho V. O socorro, o socorrido e o socorrer: cuidados em enfermagem de emergência. Rio de Janeiro: Escola de Enfermagem Anna Nery; 1999.

2. Fiocruz. Dados Radis no 20. Rio de laneiro: Fiocruz; 1996.

3. Instituto Brasileiro de Geografia e Estatística. Censo demográfico 1980, 1991 e 2000 e contagem da população 1996. Rio de Janeiro; 2000. [citado em II mar 2007]. Disponível em : http:/ /www.ibge.gov.br/

4. Certeau M. A invenção do cotidiano: artes de fazer. Petrópolis: Vozes; 1996.

5. Coelho MJ. As mil e uma maneiras de cuidar em enfermagem: a construção do conhecimento de cuidar em enfermagem e a sua aplicação no cotidiano assistencial -CNPQprocesso $521589 /$ 97-5. Relatório Final de Pesquisa. Rio de Janeiro: EEAN; 2004.

6. Coelho MI. A (in)visibilidade dos cuidados de enfermagem: um ensaio das (re)ações dos cuidados de enfermagem expressos no corpo dos clientes hospitalizados" processo 478920/200 I 6 demanda 05/200 I modalidade APQ. Relatório Final de Pesquisa cuidar/cuidados de Enfermagem Diretório dos Grupos

de Pesquisa no Brasil ref. UFRJ 0I I7. Rio de Janeiro: EEAN 2001 .

7. Coelho MI, Pereira A. Projeto Integrado (PQI/CAPES EEAN/ UFRJ e EEUFBa): mil e uma maneiras de cuidar. Rio de Janeiro: EEAN; 2004.

8. Coelho MJ. Maneiras de cuidar Enfermagem. Rev Bras Enferm 2006; 59(6): 745-5 I

9. Conselho Federal de Enfermagem. Rio de Janeiro; 2007. [citado em 10 abr 2007]. Disponível em: http://www.portal cofen.com.br/

10. Population Reference Bureau. [cited 2007 jan 20]. Available from: http://www.prb.org

11. Haddad AE, Pierantoni CR, Ristoff D, Xavier Moraes, Giolo I, Silva LB. A trajetória dos cursos de graduação na saúde 1991 2004. Brasília: INEP; 2006.

12. Cecagno D, Soares DC, Siqueira $\mathrm{HCH}$, Cecagno S. Incubadora de aprendizagem: uma nova forma de ensino na Enfermagem/ saúde. Rev Bras Enferm 2006; 59(6): 808-II.

13. Hospital Rio. Informativo de entidades representativas dos hospitais e clinicas do Rio de Janeiro. Especial SUS 2007; 9(75). 